reported in our recent paper (Collishaw et al, 2004). Controlling for childhood epilepsy/neurological problems did not reduce group differences in adult depressed affect (model adjusted only for gender: $\mathrm{OR}=2.84,95 \%$ CI 1.7-4.9, $P<0.001$; model adjusted for gender and childhood neurological problems/epilepsy: $\mathrm{OR}=2.79$, $95 \%$ CI $1.6-4.8, P<0.001)$. This is in contrast to the partial mediating effect of controlling for childhood social adversity (Maughan et al, 1999; Collishaw et al, 2004) and the almost complete mediating effect of additional controls for adult ill health and adult social adversity (Collishaw et al, 2004).

We cannot rule out completely the possibility that some other unmeasured third factor is confounded with social adversity and could explain our findings. We also acknowledge that specific biological factors may be of particular importance for understanding affective problems in some individuals with mild learning disability. Nevertheless, when assessed in an unselected general population cohort such as the NCDS, social factors and adult health do appear to have an important contribution to depressed mood among people with mild learning disability.

Collishaw, S., Maughan, B. \& Pickles, A. (2004) Affective problems in adults with mild learning disability: the roles of social disadvantage and ill health. British Journal of Psychiatry, 185, 350-351.

Maughan, B., Collishaw, S. \& Pickles, A. (1999) Mild mental retardation: psychosocial functioning in adulthood. Psychological Medicine, 29, 35I-366.

S. Collishaw, B. Maughan Box Number PO46, Institute of Psychiatry, 16 De Crespigny Park, London SE5 8AF, UK. E-mail: s.collishaw@iop.kcl.ac.uk

A. Pickles School of Epidemiology and Health Science, and Centre for Census and Survey Research, University of Manchester, UK

\section{Prevalence of dementia}

We thank Dr Varghese (2005) for his letter regarding our article (Shaji et al, 2005). Lack of education is a potent predictor of poor performance across many items of the Chinese version of the Mini-Mental State Examination (MMSE; Katzman et al, 1988). There was no significant difference between total MMSE scores of those who were illiterate and those who were literate in the pilot study conducted with the Malayalam adaptation of the MMSE. Hence it was decided to use the same score for both groups.
We identified 55 cases of dementia among 327 people who scored at or below the cut-off on the MMSE. The one case identified from the $10 \%$ of the negatively screened population was counted as one among the ten cases in the negatively screened population of 1607 (i.e. 65 cases in 1934 people aged 65 years and above).

The assessment of risk factors based on retrospective accounts of the carers and an inadequate number of controls for calculating the odds ratios can be considered methodological limitations of the study. The prevalence of dementia increases proportionately with age $\left(\chi^{2}=40.29\right.$, d.f. $=5$, $P<0.001)$. This $\chi^{2}$ value was not given in the text. The number of patients with Alzheimer's disease was 30 . The error in the article is regretted.

Katzman, R., Zhang, M.Y., Ouang-Ya-Ou, et al (1988) A Chinese version of the Mini-Mental State Examination; impact of illiteracy in a Shanghai dementia survey. Journa of Clinical Epidemiology, 41, 97I-978.

Shaji, S., Bose, S. \& Verghese, A. (2005) Prevalence of dementia in an urban population in Kerala, India. British Journal of Psychiatry, 186, 136-140.

Varghese, S. T. (2005) Dementia prevalence (letter) British Journal of Psychiatry, 186, 542.

S. Shaji Bethsada Hospital, Vengola PO, Pin 683 554, Ernakulam District, Kerala, India. E-mail: sdrshaji@rediffmail.com

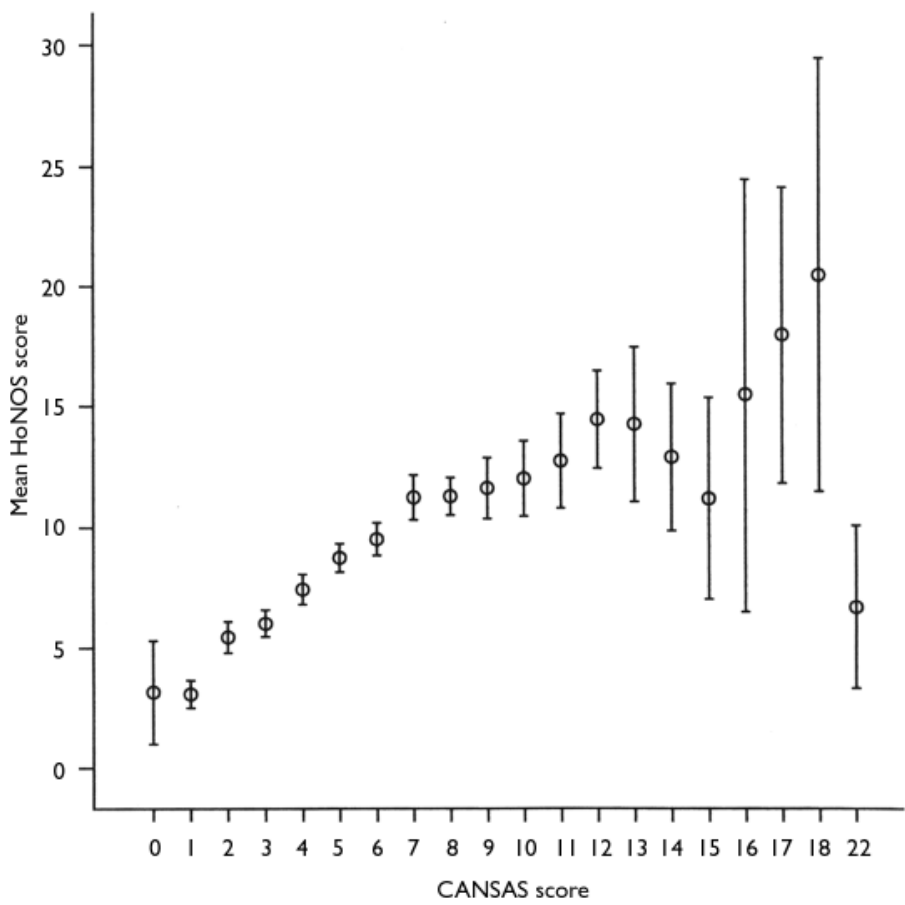

Fig. I Comparison of 1000 pairs of CANSAS and HoNOS scores. Bars represent two standard errors above and below the mean. CANSAS, Camberwell Assessment of Need Short Appraisal Schedule; HoNOS, Health of the Nation Outcome Scale.

\section{Measures for mental health outcomes}

I was very interested to read the article by Salvi et al (2005) on choosing the measure for mental health outcome assessments. Readers might be interested in a comparison of the Camberwell Assessment of Need Short Appraisal Schedule (CANSAS; Phelan et al, 1995) and Health of the Nation Outcome Scale (HoNOS; Wing et al, 1998) scores. One thousand pairs of HoNOS and CANSAS scores were recorded by four trainees and myself. Figure 1 shows the means with standard errors of the HoNOS values associated with each CANSAS score.

The higher CANSAS scores (13-22) were not encountered very often and accounted for only $3.5 \%$ of scores. The large standard errors are because some of the CANSAS scores occurred infrequently.

HoNOS and CANSAS scores are related in the lower CANSAS range of 1-8, the most common range, accounting for $79 \%$ of the scores. Up to a CANSAS score of $12(n=955)$ there is a reasonably close correlation with the HoNOS scores. The Spearman coefficient is 0.564 , indicating that the correlation is significant at the 0.01 level (two-tailed).

The use of CANSAS is becoming established in Lothian mental health services. CANSAS is very useful as a needs assessment tool for individual patients. Its face 
validity as an outcome measure is not as good as that of the Threshold Assessment Grid (Slade et al, 2000), Global Assessment of Functioning (Jones et al, 1995) or HoNOS. This correlation exercise confirms that it can be used as an outcome measure with reasonable validity. It is useful in terms of consultant appraisal discussions, evaluation of workload of community and ward mental health teams and local and regional assessment of outcomes in different patient groups. Given the above correlation, benchmarking is also possible with other services, especially in England, where HoNOS is established. The conclusions of Salvi et al (2005) in the last paragraph of their article are absolutely valid.

Given the great difficulty in implementing and coordinating any single outcome assessment, I hope that the above comparison of CANSAS and HoNOS scores, in combination with the results of Salvi et al (2005), will assist those running mental health services.

Jones, S. H., Thornicroft, G., Coffey, M., et al (1995) A brief mental health outcome scale: reliability and validity of the Global Assessment of Functioning (GAF). British Journal of Psychiatry, 166, 654-659.

Phelan, M, Slade, M., Thornicroft, G., et al (1995) The Camberwell Assessment of Need (CAN): the validity and reliability of an instrument to assess the needs of people with severe mental illness. British Journal of Psychiatry, 167, 589-595.

Salvi, G., Leese, M. \& Slade, M. (2005) Routine use of mental health outcome assessments: choosing the measure. British Journal of Psychiatry, 186, 146-152.

Slade, M., Powell, R., Rosen, A., et al (2000)

Threshold Assessment Grid (TAG): the development of a valid and brief scale to assess the severity of mental illness. Social Psychiatry and Psychiatric Epidemiology, 35, 78-85

Wing, J. K., Beevor, A. S., Curtis, R. H., et al (1998) Health of the Nation Outcome Scales (HoNOS): research and development. British Journal of Psychiatry, 172, II-18.

R. J. Craig Rosslynlee Hospital, Roslin, Midlothian EH25 9QE,UK. E-mail: James.Craig@|pct.scot.nhs.uk

\section{Publication of case reports}

Several letters advocating the reinstatement of case reports in the Journal have been published recently (Williams, 2004; Enoch, 2005). I believe that it would be useful to make a distinction between two substantially different kinds of such reports. The first group includes discussions of challenging cases with difficult clinical implications and interesting phenomenological descriptions, with the only aim to improve the readers' diagnostic and therapeutic skills. Typical examples are the 'Grand Rounds' that used to be published in the $B M J$. I agree with $\mathrm{Dr}$ Enoch and $\mathrm{Dr}$ Williams' point of view and I would personally welcome the publication of these case reports in the Journal.

However, another group of reports have a substantially different objective. Their aim is to allow clinicians to share their anecdotal experience of unusual outcomes in clinical practice. These reports are a self-selected group of unlikely cases because only 'man bites dog' stories reach publication. The conclusions of sophisticated randomised trials with good statistical analyses are difficult enough to interpret because of biases such as unmasking, file drawer problems, etc. Anecdotal care reports can be confusing and misleading because the subjective data are often interpreted as objective, creating even more noise where the signal is already faint. The publication of a one-off case report of an adverse effect can profoundly influence clinical practice on the basis of a freak event. Infamous examples include the widely followed recommendation not to use haloperidol and lithium in combination (Cohen \& Cohen, 1974) and the reluctance to use intravenous thiamine for the prevention of Korsakoff syndrome on the basis of a few reports of adverse reactions (Thomson \& Cook, 1997). The cases of the hundreds of thousands of people who have been safely and successfully treated with these medications are not published because no one wants to state the obvious. I believe that the past editor's decision to move on from publishing this latter group of case reports was extremely wise.

Cohen, W. J. \& Cohen, N. H. (1974) Lithium carbonate haloperidol, and irreversible brain damage. JAMA, 230 , 1283-1287.

Enoch, M. D. (2005) Case reports (letter). British Journal of Psychiatry, 186, 169.

Thomson, A. D. \& Cook, C. C. (1997) Parentera thiamine and Wernicke's encephalopathy: the balance of risks and perception of concern. Alcohol and Alcoholism, 32, 207-209.

Williams, D. D. R. (2004) In defence of the case report (letter). British Journal of Psychiatry, 184, 84.

M. Procopio Priory Hospital, Hove BN3 4FH, UK. E-mail: marcoprocopio00@hotmail.com

Editor's response: We do publish case reports if they have, or could have, important general implications. The paper by Boddaert and her colleagues in this issue (Boddaert et al, 1995) is a good example of this.
Boddaert, N., Barthélémy, C., Poline, J.-B., et a

(2005) Autism: functional brain mapping of exceptional calendar capacity. British Journal of Psychiatry, 187, 83-86.

Peter Tyrer Editor, British Journal of Psychiatry, 17 Belgrave Square, London SWIX 8PG, UK. E-mail: bjp@rcpsych.ac.uk

\section{ECT for acute mania}

In his excellent review of the management of acute mania, Professor Keck does not mention an additional form of available treatment, no doubt because it is archaic and anecdotal.

In the early 1950 s, when the only drugs available to treat mania were paraldehyde and barbiturates, patients were ill for months, and sometimes even died of exhaustion. In those days 'electroplexy' was given for everything, but a standard course of treatment of seven sessions of electroconvulsive therapy (ECT) over 3 weeks proved ineffective in manic patients. However, it became apparent that ECT applied twice daily, over 3 or at the most 4 days, usually brought the manic attack to an end.

I last used this treatment over 20 years ago, in circumstances where prompt restoration to health was vital. It was completely successful. The real difficulty was in obtaining anaesthetic cover twice daily. In drug-resistant cases such an approach might still have a place, with considerable savings in the time spent in hospital.

Keck, P. E. Jr (2003) The management of acute mania. $B M J$, 327, 1002-1003.

A. C. Gibson Royal College of Physicians, 9 Queen Street, Edinburgh EH2 IJQ, UK. E-mail: alangibson@transformingeducation.co.uk

\section{Variations in involuntary commitment in the European Union}

The recent article by Salize \& Dressing (2004) reported that frequencies of compulsory admissions vary remarkably among countries in the European Union, from 6 per 100000 citizens in Portugal to 218 per 100000 in Finland. These findings are not surprising given the large differences in the laws, mental health acts, and legal instruments of the countries but they are astonishing given the much smaller differences in psychiatric morbidity. These differences show that the number of involuntary admissions is a result of a complex set of still poorly understood legal, political, economic, social and multiple other factors 